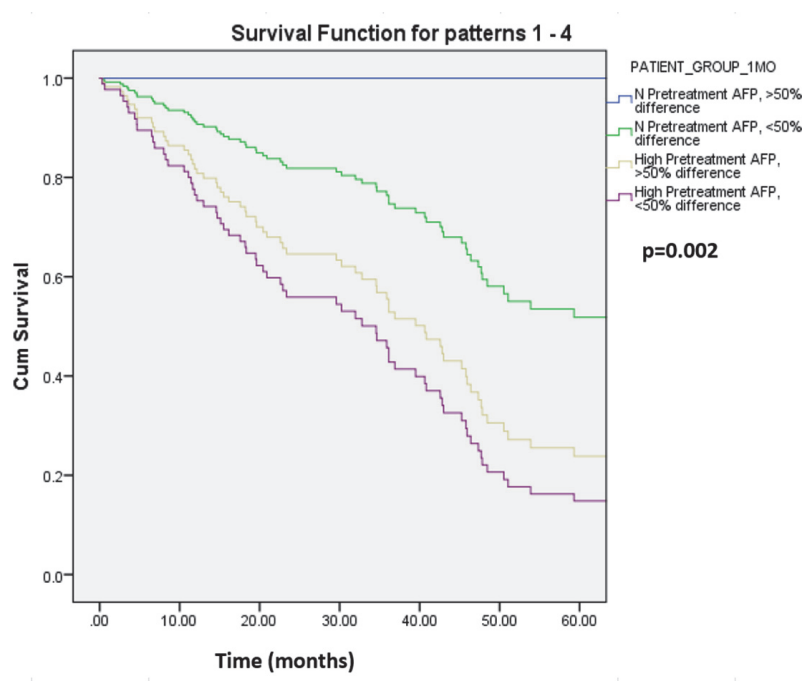

Abstract IDDF2021-ABS-0088 Figure 1 Kaplan-Meier curve for overall survival of HCC patients based on pre-treatment AFP and posttreatment AFP response $\geq 50 \%$ at 1 -month after RFA

analyzed, pre-treatment $\mathrm{AFP}<10 \mathrm{ng} / \mathrm{ml}$ and post-treatment AFP response $\geq 50 \%$ at 1 month yielded the best estimated 5 -year survival $(p=0.002)$ - (IDDF2021-ABS-0088 Figure 1. KaplanMeier Curve for Overall Survival of HCC Patients based on Pre-treatment AFP and Post-treatment AFP response $\geq 50 \%$ at 1 month after RFA).

Conclusions Pre-treatment $\mathrm{AFP}<10 \mathrm{ng} / \mathrm{ml}$, 6-months post-treatment AFP response $\geq 50 \%$ with normal AFP and 1- and 3months post-treatment AFP response $\geq 50 \%$ with high AFP were predictors of better OS. AFP $<10 \mathrm{ng} / \mathrm{mL}$ at any time during 1-,3- or 6-months after RFA was an important predictor of better prognosis. The best prognosticator for OS was a patient with pre-treatment $\mathrm{AFP}<10 \mathrm{ng} / \mathrm{ml}$ and post-treatment AFP response $\geq 50 \%$ at 1 month.

\section{IDDF2021-ABS-0089 PRIMARY NORFLOXACIN PROPHYLAXIS REDUCES THE INCIDENCE OF INFECTIONS IN SEVERE ALCOHOLIC HEPATITIS-A DOUBLE-BLIND, RANDOMIZED CONTROLLED STUDY}

Anand Kulkarni*, Sowmya Iyengar, Syeda Fatima, Mithun Sharma, PN Rao, D Nageshwar Reddy. Asian Institute of Gastroenterology, India

\subsection{6/gutjnl-2021-IDDF.86}

Background Steroid therapy is the standard of care for severe alcoholic hepatitis (sAH) at most centres. However, steroids are associated with an increased risk of infections. Therefore, we aimed to compare the outcomes of patients receiving concomitant norfloxacin (NFX) with steroids in patients with sAH.

Methods In this double-blind, randomized study, acute-onchronic liver failure (ACLF) patients without sepsis, hepatic encephalopathy, or SBP were assigned to receive oral NFX $400 \mathrm{mg}$ or matched placebo (PBO) once daily for 30 days along with standard medical therapy. 143 ACLF patients were included (CTRI/2019/10/021548). On subgroup analysis, 20 in the NFX group and 13 patients in the PBO group received concomitant steroid therapy for sAH. The primary objective was to assess the incidence of infections at days 30, 90, and

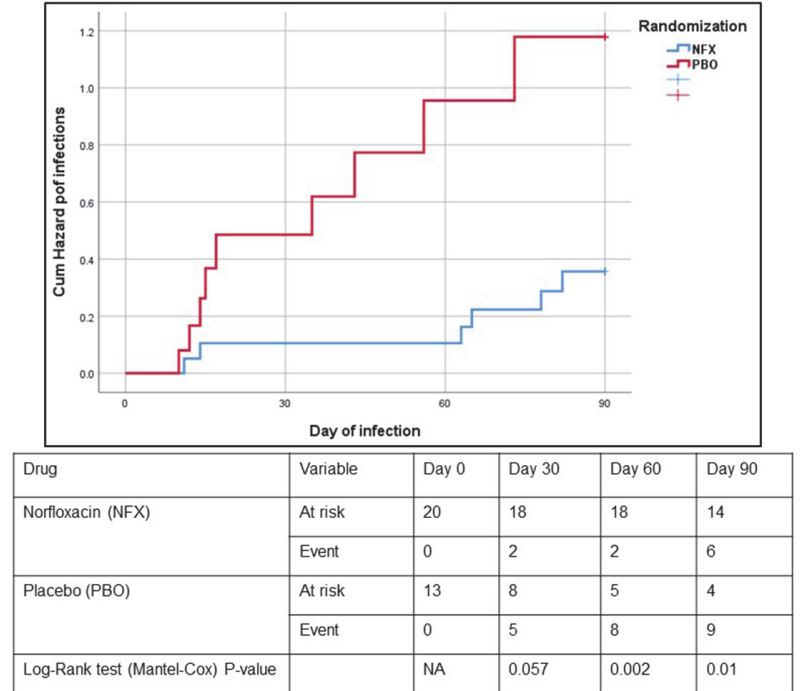

Abstract IDDF2021-ABS-0089 Figure 1

the secondary was to evaluate the transplant-free survival (TFS) at days 30, 90.

Results Baseline characteristics were similar in both the groups (MELD-NFX-27.8 \pm 3.79 vs. PBO-29.62 $\pm 4.66 ; \mathrm{P}=0.229$ ). The incidence of infection was lower with NFX (10\%) than PBO $(38.5 \%)$ at day $30(\mathrm{P}=0.066)$. The incidence of infection at day 90 was lower with NFX (30\%) than PBO (69.2\%) $(\mathrm{P}=0.027)$ (IDDF2021-ABS-0089 Figure 1. Kaplan Meier analysis of infection incidence at days 30,60 and 90). TFS was similar in both groups at day 30 (NFX-85\% vs. PBO-77\%; Log-Rank test: $\mathrm{P}=0.524)$. However, TFS at day 90 was better with NFX (85\%) than placebo $(53.8 \%)$ (Log-Rank test: $\mathrm{P}=0.059)$. The most common source of infection was the urinary tract (NFX - 3 vs. Placebo-4). E. coli was the commonly isolated organism in both groups. Candida albicans were isolated in two patients in NFX group, while none in the PBO group developed fungal infection. Sepsis was the common cause of mortality (NFX-2; PBO-4). Further, one patient in NFX group succumbed to post-TIPS liver failure (for variceal bleed). There were no drug-related adverse events in this subgroup of patients.

Conclusions Primary norfloxacin prophylaxis is safe and effective in reducing infections in patients with sAH receiving steroid therapy.

IDDF2021-ABS-0092
A DIAGNOSTIC TEST META-ANALYSIS
EVALUATING THE PERFORMANCE
OF IMAGING-BASED AND BLOOD
BIOMARKER-BASED ASSESSMENT
TOOLS FOR FIBROSIS AFTER LIVER
TRANSPLANTATION

${ }^{1}$ Cheng Han Ng* ${ }^{*}$ Xiong Chang Lim, ${ }^{1}$ Darren Jun Hao Tan, ${ }^{1} J i e$ Ning Yong, ${ }^{1}$ Nicholas Syn, ${ }^{1}$ Gwyneth Shook Ting Soon, ${ }^{1}$ Eunice Xiang Xuan Tan, 'Yock Young Dan, ${ }^{1}$ Mark Dinesh Muthiah, ${ }^{2}$ Mohammad Shadab Siddiqui. 'Yong Loo Lin School of Medicine, Singapore; ${ }^{2}$ Vierginea Commonwealth University, Virginia, USA

\subsection{6/gutjnl-2021-IDDF.87}

Background Assessing fibrosis after liver transplant (LT) remains a core concern and can suggest the presence of de novo nonalcoholic fatty liver disease or recurrence after 
transplant. While biopsy remains the gold standard for assessing fibrosis, the acceptability of periodic fibrosis by patients remains a barrier for routine use. Noninvasive tests (NITs) can be used to assess fibrosis after LT. However, the diagnostic accuracy of these tests remains unknown after transplant. Hence, we seek to evaluate the accuracy of NIT after LT.

Methods We conducted a systematic search of the literature in Medline and Embase on 15 June 2021. Articles relating to the use of vibration-controlled transient elastography (VCTE), 2D shear wave elastography (2DSWE), magnetic resonance elastography (MRE), acoustic radiation force impulse (ARFI), fibrotest, AST to Platelet Ratio Index (APRI), and the Fibrosis-4 Index (FIB-4) were included in the study. Evaluation of diagnostic accuracy was done with a bivariate binomial model, and comparisons between tests were established with likelihood ratio estimates.

Results In total, 1,829 individuals were assessed for fibrosis using biopsy after liver transplant. 644 individuals had fibrosis of $\mathrm{F} 2 \geq(37.01 \%, \mathrm{CI}: 28.78$ - 46.07) F3 $\geq(16.85 \%$, CI: 10.92 - 25.08), and F4 was and (9.93\%, CI: 7.42 - 13.16) respectively. The results are summarized in (IDDF2021-ABS-

\begin{tabular}{|c|c|c|c|c|c|c|c|}
\hline Diagnostic Tool & No. of Studies & $\mathbf{N}$ & Sensitivity & Specificity & DOR & Positive LR & Negative LR \\
\hline \multicolumn{8}{|c|}{ Significant Fibrosis (F2-F4) } \\
\hline \multirow[t]{2}{*}{ 2DSWE } & 2 & 16 & $0.745(0.507-0.893)$ & $0.706(0.557-0.821)$ & $7.007(1.913-25.667)$ & $2.531(1.450-4.419)$ & $0.361(0.157-0.825)$ \\
\hline & & 7 & & & & & \\
\hline \multirow[t]{2}{*}{ APRI } & 6 & 53 & $0.702(0.624-0.769)$ & $0.747(0.651-0.823)$ & $6.943(3.628-13.290)$ & $2.771(1.886-4.072)$ & $0.399(0.296-0.538)$ \\
\hline & & 8 & & & & & \\
\hline \multirow[t]{2}{*}{ ARFI } & 5 & 50 & $0.864(0.772-0.922)$ & $0.753(0.622-0.849)$ & 19.292(9.309-39.984) & $3.495(2.241-5.450)$ & $0.181(0.108-0.303)$ \\
\hline & & 7 & & & & & \\
\hline \multirow[t]{2}{*}{ FIB-4 } & 2 & 14 & $0.657(0.305-0.893)$ & $0.729(0.435-0.904)$ & $5.153(1.912-13.883)$ & $2.423(1.291-4.538)$ & $0.470(0.222-0.996)$ \\
\hline & & 3 & & & & & \\
\hline Fibrotest & 2 & 114 & $0.684(0.522-0.811)$ & $0.403(0.211-0.631)$ & $1.464(0.462-4.639)$ & $1.146(0.744-1.766)$ & $0.783(0.379-1.618)$ \\
\hline MRE & 2 & 83 & $0.586(0.374-0.771)$ & $0.813(0.347-0.973)$ & $6.135(0.878-42.852)$ & $3.126(0.628-15.567)$ & $0.510(0.306-0.848)$ \\
\hline \multirow[t]{2}{*}{ VCTE } & 10 & 84 & $0.796(0.714-0.860)$ & $0.838(0.785-0.880)$ & $20.18(11.61-35.07)$ & $4.908(3.630-6.636)$ & $0.243(0.170-0.348)$ \\
\hline & & 1 & & & & & \\
\hline \multicolumn{8}{|c|}{ Advance Fibrosis (F3-F4) } \\
\hline \multirow[t]{2}{*}{ APRI } & 2 & 14 & $0.488(0.314-0.637)$ & $0.790(0.673-0.872)$ & $3.572(1.516-8.418)$ & $2.317(1.313-4.090)$ & $0.649(0.469-0.897)$ \\
\hline & & 3 & & & & & \\
\hline \multirow[t]{2}{*}{ ARFI } & 3 & 35 & $0.871(0.629-0.964)$ & $0.832(0.634-0.934)$ & $28.142(7.135-110.996)$ & $4.766(2.179-10.424)$ & $0.155(0.049-0.491)$ \\
\hline & & 7 & & & & & \\
\hline \multirow[t]{2}{*}{ FIB-4 } & 3 & 39 & $0.612(0.348-0.823)$ & $0.718(0.484-0.874)$ & $4.016(1.434-11.25)$ & $2.170(1.170-4.026)$ & $0.540(0.305-0.957)$ \\
\hline & & 3 & & & & & \\
\hline MRE & 3 & 114 & $0.864(0.652-0.955)$ & $0.728(0.629-0.809)$ & $16.97(4.619-62.37)$ & $3.178(2.188-4.617)$ & $0.187(0.065-0.540)$ \\
\hline \multirow[t]{2}{*}{ VCTE } & 3 & 32 & $0.796(0.652-0.890)$ & $0.913(0.840-0.954)$ & $44.77(18.79-88.49)$ & $9.122(5.086-16.36)$ & $0.224(0.128-0.392)$ \\
\hline & & 6 & & & & & \\
\hline \multicolumn{8}{|l|}{ Cirrhosis (F4) } \\
\hline \multirow[t]{2}{*}{ APRI } & 2 & 23 & $0.944(0.693-0.992)$ & $0.427(0.364-0.494)$ & $12.683(1.658-96.992)$ & $1.649(1.405-1.935)$ & $0.130(0.019-0.879)$ \\
\hline & & 8 & & & & & \\
\hline \multirow[t]{2}{*}{ VCTE } & 5 & 50 & $0.919(0.746-0.978)$ & $0.930(0.836-0.972)$ & $150.0(36.59-615.0)$ & $13.10(5.519-31.07)$ & $0.087(0.026-0.296)$ \\
\hline & & 7 & & & & & \\
\hline
\end{tabular}

Sensitivity and Specificity Comparison in Significant Fibrosis (F2-F4)
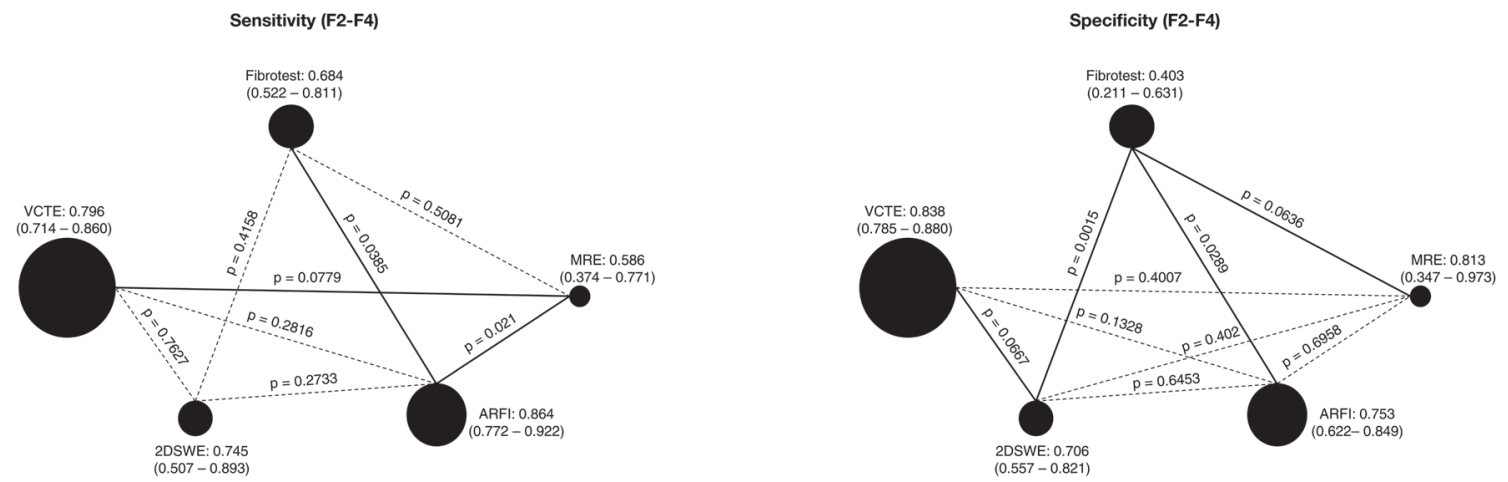

Abstract IDDF2021-ABS-0092 Figure 1 


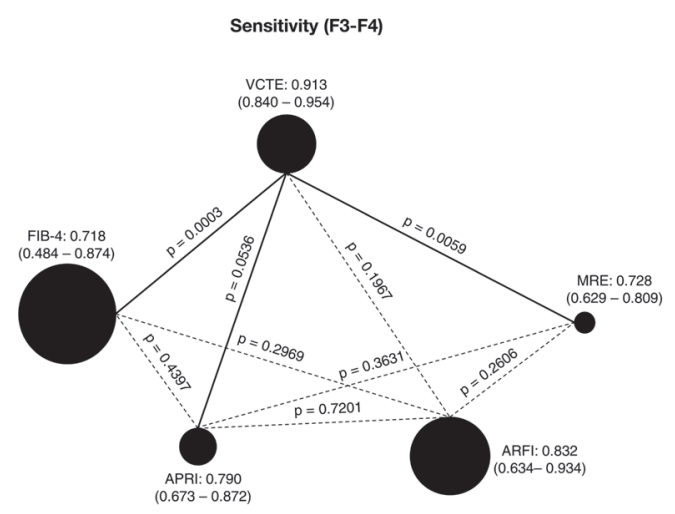

Abstract IDDF2021-ABS-0092 Figure 2

0092 Table 1). In brief, AFRI and VCTE provided sufficient diagnostic accuracy at F2 and F3. VCTE was additionally suited for F4 diagnosis. Blood-based NIT was found to be an inaccurate measure of fibrosis. When comparisons were conducted between NITs, there was no significant difference between VCTE and AFRI at F2 and F3 in both sensitivity and specificity (IDDF2021-ABS-0092 Figure 1, IDDF2021ABS-0092 Figure 2). Blood-based NIT was inferior to VCTE and AFRI.

Conclusions To our knowledge, our study is the first systematic evaluation of NITs after transplant. The diagnostic accuracy suggests that VCTE and ARFI should be employed as measures of fibrosis after LT. The presence of thrombocytopenia after LT results in the reduced accuracy of FIB-4 and ARFI. More studies are required to assess other measures of NITs, including MRE.

\section{IDDF2021-ABS-0095 A META-ANALYSIS AND SYSTEMATIC REVIEW ON THE GLOBAL PREVALENCE, RISK FACTORS AND OUTCOMES OF CORONARY ARTERY DISEASE IN LIVER TRANSPLANT RECIPIENTS}

${ }^{1}$ Cheng Han Ng*, 'Jieling Xiao, 'Jie Ning Yong, 'Nicholas Syn, 'Wen Hui Lim, 'Darren Jun Hao Tan, ${ }^{1}$ En Ying Tan, ${ }^{1}$ Daniel Huang, ${ }^{2}$ Raymond Wong, ${ }^{2}$ Nicholas Chew, ${ }^{1}$ Eunice Xiang Xuan Tan, ${ }^{3}$ Mazen Noureddin, ${ }^{1}$ Mark Dinesh Muthiah, ${ }^{4}$ Mohammad Shadab Siddiqui. ${ }^{1}$ Yong Loo Lin School of Medicine, Singapore; ${ }^{2}$ Department of Cardiology, National University Heart Centre, National University Hospital, Singapore; ${ }^{3}$ Cedars-Sinai Fatty Liver Program, Division of Digestive and Liver Diseases, Department of Medicine, Comprehensive Transplant Center, Cedars-Sinai Medical Center, Los Angeles, USA; ${ }^{4}$ Division of Gastroenterology, Hepatology and Nutrition, Department of Internal Medicine, Virginia Commonwealth University, Richmond, Virginia, USA

\subsection{6/gutjnl-2021-IDDF.88}

Background The changing demographics of waitlisted liver transplantation (LT) patients and shift in etiology of liver disease result in increasing prevalence of coronary artery disease (CAD), which can impact post-LT outcomes. This study aims to evaluate the prevalence of CAD among potential LT recipients, describe the risk factors of $\mathrm{CAD}$ and investigate the impact of pre-LT CAD on post-LT outcomes.

Methods Two electronic databases, MEDLINE and EMBASE, were searched for articles describing the CAD in pre-LT patients. Articles that describe the prevalence, risk factors and outcomes of pre-LT patients diagnosed with CAD were

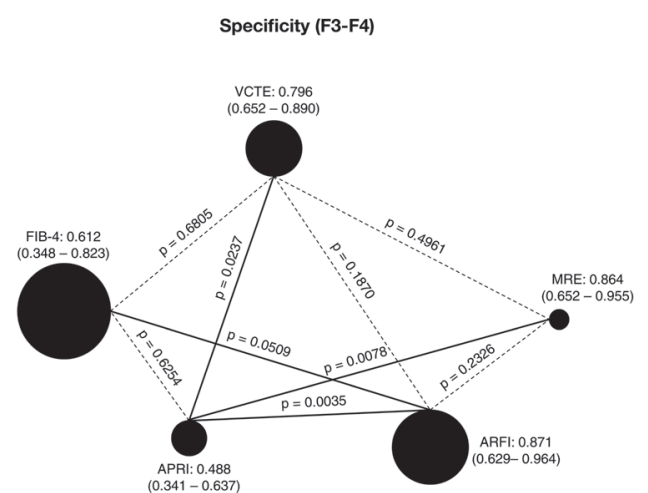

included. Generalized linear mixed model (GLMM) and Clopper-Pearson intervals to stabilize the variance were used to tabulate the prevalence of CAD in pre-LT patients and outcomes post-LT. The prevalence of CAD in pre-LT patients was stratified across different regions and countries of varying income levels. Risk factors of CAD and outcomes were analyzed with regression or pairwise analysis.

Results Total of 39 articles were included. The pooled prevalence of 15,880 patients from 34 studies diagnosed with CAD before LT was 15.88\% (CI: 9.81\% - 24.69\%, IDDF2021-ABS-0095 Figure 1. Screening Method of CAD before Liver Transplant). LT candidates in high-income countries had markedly higher odds of CAD (OR: 10.5; CI: 8.12 - 13.7; p < 0.0001) compared to patients in middle-income countries. Age, male, presence of diabetes, hypertension, hyperlipidemia, smoking, non-alcoholic steatohepatitis, hepatitis B and hepatocellular carcinoma were significantly associated with CAD. CAD diagnosis prior to LT was a significant risk factor for overall mortality (OR: 1.42; CI: $1.40-1.43$; $\mathrm{p}=0.0140$ ) and cardiac-related mortality (OR: 1.18; CI: $1.10-1.27 ; \mathrm{p}=0.0347)$ post-LT. North America had the highest pooled prevalence of CAD in pre-LT patients at 23.61\% (CI: 14.07\% - 36.83\%) amongst South America, Europe, Middle east, Asia and Oceania. 69\% of the included studies utilized non-invasive stress tests and invasive coronary angiograms in their pre-LT cardiac assessment (IDDF2021ABS-0095 Figure 2. Global Prevalence of CAD before Liver Transplant).

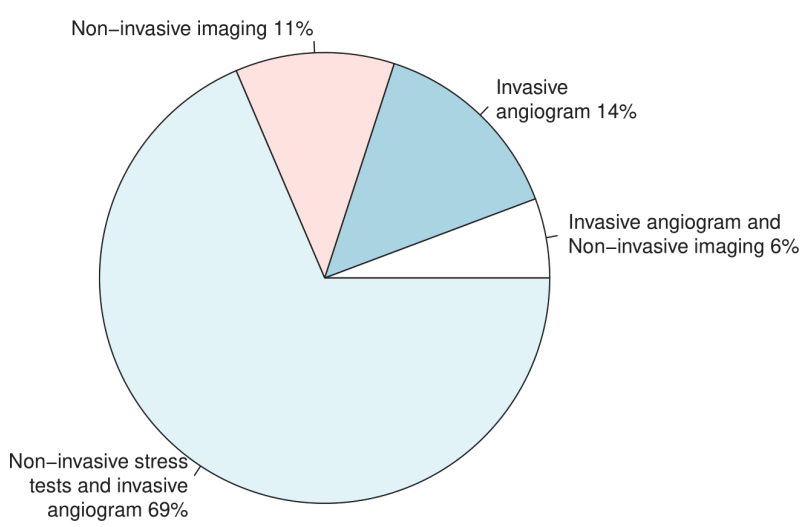

Abstract IDDF2021-ABS-0095 Figure 1 